\title{
First report of the occurrence of Myrothecium verrucaria in watermelon seeds from India
}

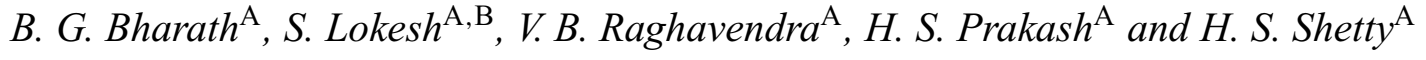 \\ ${ }^{\text {A }}$ Department of Studies in Applied Botany, Seed Pathology and Biotechnology, University of Mysore, Manasagangotri, \\ Mysore-570 006, Karnataka, India. \\ ${ }^{\mathrm{B}}$ Corresponding author. Email: boramma@rediffmail.com
}

Abstract. Watermelon (Citrullus lanatus) is known to be affected by a variety of both seed-borne and soil-borne fungi. In routine screening of watermelon seed samples, sporodochia of Myrothecium verrucaria were observed. The fungus was isolated and the spore suspension was inoculated onto healthy seedlings of watermelon. The resulting symptoms confirmed Koch's postulates.

Watermelon [Citrullus lanatus (Thunb.) Matsum and Nakai], is a commercial crop grown for its fleshy, refreshing fruits. It is widely cultivated in tropical and subtropical countries throughout the world. The crop is susceptible to a variety of fungal, bacterial and viral diseases at various stages of its growth (Sharma and Khan 1991; Roberts and Kucharck 2003). In this study, seeds of two popular varieties, Arka Manik and Sugar Baby, were incubated on wet blotters following the standard procedures of ISTA. Routine examination of the seeds showed a high incidence of a pathogenic fungus Myrothecium verrucaria (Albertini \& Schewein) Detmar ex Fr. Infected seeds showed many cushioned, light green sporodochia with a white hairy margin. $M$. verrucaria showed hyaline to very light green coloured, aseptate spores with acute ends (Fig. 1A, B). Myrothecium species generally cause round dark brown leaf spots in cucurbits, which on later stages coalesce to form blighted areas on the leaves (Belisario et al. 1999).

To evaluate the pathogenicity of the fungus, 10-day-old seedlings raised in wet sterilised sand beds in plastic trays were collected without damaging the roots. The seedlings were inoculated by dipping the roots in the freshly harvested spore suspension $\left(1 \times 10^{6}\right.$ spores $\left./ \mathrm{mL}\right)$. The spore suspension was obtained from 8-day-old sporulated colonies of the $M$. verrucaria watermelon isolate, which was grown on potato dextrose agar medium at $22 \pm 2{ }^{\circ} \mathrm{C}$. The inoculated seedlings were then transplanted to pots containing sterilised moist soil and sand in a 1:1 ratio. Seedlings not inoculated with the fungal spore suspension, but transplanted and maintained in similar conditions were used as the control. For both treatments, the pots were maintained in the greenhouse at $25 \pm 2^{\circ} \mathrm{C}$ under diffused light conditions.
In another experiment, the seeds were inoculated with the same isolate of the fungus by rolling on 10-day-old sporulating colonies and were sown in the sterilised wet soil: sand bed as described above. On the 10th day after inoculation, the seedlings were carefully observed for the occurrence of disease symptoms. All the seedlings showed damping-off. Necrosis of cotyledonary leaves was also observed 7 days after inoculation, and was found to be initiated at the tip and marginal portions of the leaves (Fig. 1C). These necrotic cotyledons were harvested from the seedlings and incubated on wet blotters in plastic plates at $22 \pm 2{ }^{\circ} \mathrm{C}$. On the fourth day of incubation, the necrotic portions showed luxuriant, well-developed light green sporodochia (Fig. 1D). The shoot portions also showed similar sporodochia (Fig. 1E), and the entire seedling showed damping-off symptoms (Fig. $1 F$ ). These sporodochia were inoculated on to PDA and incubated for a period of 8 days. The 8-day-old sporulating cultures were used to inoculate the watermelon seeds as described above. The inoculated seeds were then sown in the sterilised wet soil: sand beds and incubated. The growing seedlings were observed for the occurrence of similar symptoms. The repetitive symptoms on the cotyledonary leaves and the occurrence of sporodochia on different parts of the seedlings proved Koch's postulates.

Yang and Jong (1995) have described the host range of $M$. verrucaria, isolated from leafy spurge. Belisario et al. (1999) have also reported the occurrence of the fungus on the muskmelon seeds under in vitro conditions. However, this study reports the only known description of the occurrence of $M$. verrucaria on the seeds of watermelon.

This study confirms that the watermelon symptoms were due to infection by $M$. verrucaria. This observation provides 

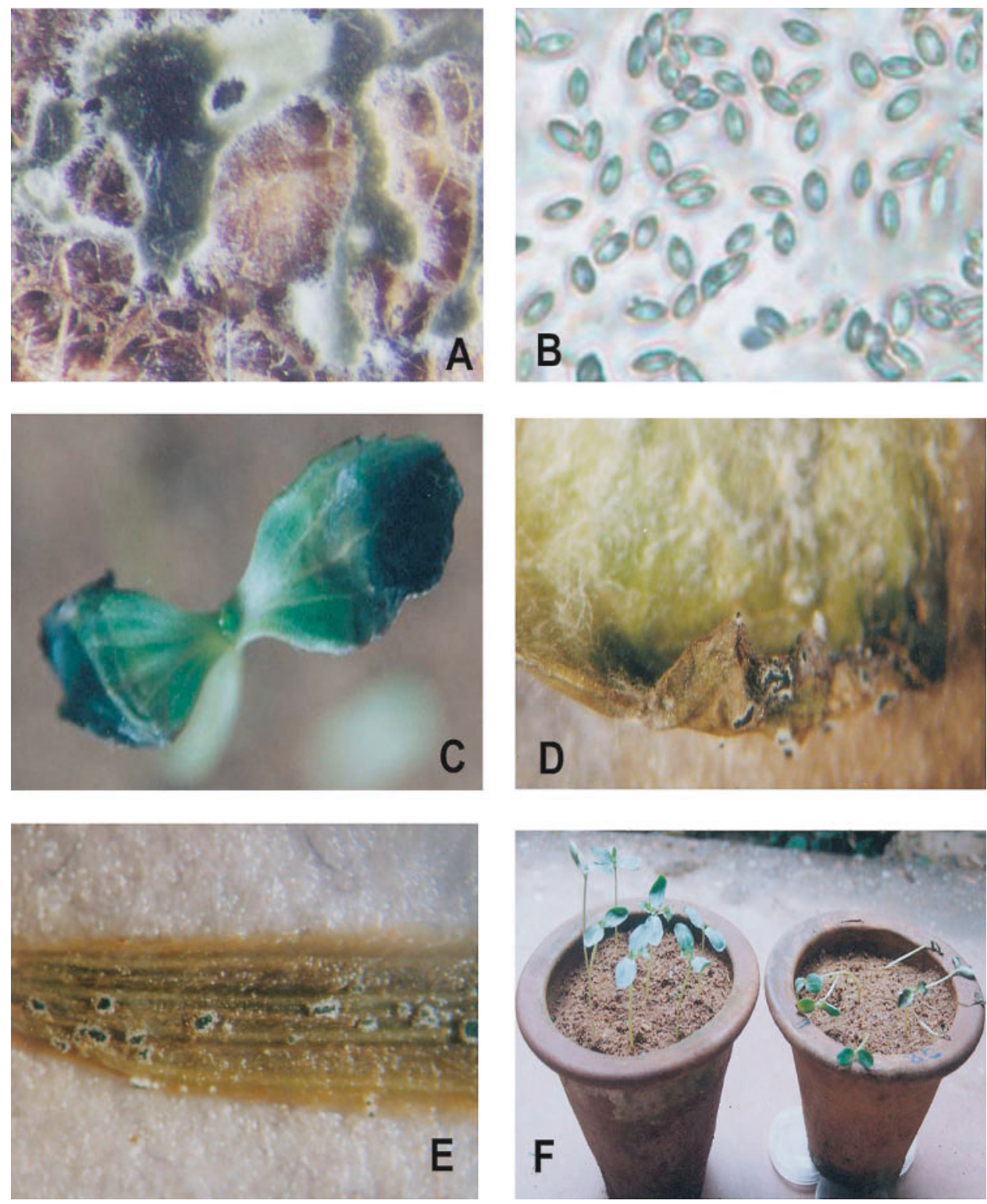

Fig. 1. (A) Myrothecium verrucaria showing sporodochia on watermelon seeds $(25 \times)$; $(B)$ Conidia of $M$. verrucaria $(40 \times) ;(C)$ Watermelon seedling showing cotyledonary necrosis due to $M$. verrucaria infection; $(D)$ A close-up view of cotyledonary necrosis with sporodochia of $M$. verrucaria $(5 \times)$; $(E)$ Decay shoot of watermelon with sporodochia of M. verrucaria $(50 \times) ;(F)$ Seedling showing damping-off due to M. verrucaria.

important information for the growth of watermelon crops: better crop growth may be achieved by the selection of seeds free from this pathogen. Seed screening may provide a complementary treatment to the use of seed treatments to control this fungal disease.

\section{References}

Belisario A, Forti E, Corazza L, Van Kesteren HA (1999) First report of Myrothecium verrucaria from muskmelon seeds. Plant Pathology 83, 589 .
Roberts R, Kucharck T (2003) 'Florida plant disease management guide: cucumber.' (Edis, Florida Cooperative Extension Service, Institute of Food and Agricultural Science, University of Florida: Gainesville, FL)

Sharma GK, Khan W (1991) Observation on occurrence and identity of powdery mildew of cucurbits in Tamil Nadu. Indian Phytopathology 48, 314-324.

Yang SM, Jong SC (1995) Host range determination of Myrothecium verrucaria isolated from leafy spurge. Plant Disease 79, 994-997.

Accepted 22 February 2006 